\title{
Thermoelectric Transport in a ZrN/ScN Superlattice
}

\author{
MONA ZEBARJADI,${ }^{1}$ ZHIXI BIAN,${ }^{1}$ RAJEEV SINGH,${ }^{1}$ ALI SHAKOURI,${ }^{1,3}$ \\ ROBERT WORTMAN, ${ }^{2}$ VIJAY RAWAT, ${ }^{2}$ and TIM SANDS ${ }^{2}$ \\ 1.-Department of Electrical Engineering, University of California, Santa Cruz, CA 95064, USA. \\ 2.-Birck Nanotechnology Center, Purdue University, West Lafayette, IN, USA. 3.- - -mail: \\ ali@soe.ucsc.edu
}

\begin{abstract}
Metal/semiconductor superlattices have the potential for a high thermoelectric figure of merit. The thermopower of these structures can be enhanced by controlling the barrier height using high-energy electron filtering. In addition, phonon scattering at interfaces can reduce the lattice contribution to the thermal conductivity. In this paper, we present theoretical and experimental studies of the thermoelectric transport in $\mathrm{ZrN} / \mathrm{ScN}$ metal/semiconductor superlattices. Preliminary measurement results show an exponential increase in the cross-plane electrical conductivity with increasing temperature, which indicates the presence of the barrier. Fit of the Boltzmann transport-based model with the data indicates a barrier height of $280 \mathrm{meV}$. The cross-plane Seebeck coefficient of the sample is also measured by combining Seebeck voltage transient measurements with the thermal imaging technique. A Seebeck coefficient of $820 \mu \mathrm{V} / \mathrm{K}$ at room temperature is extracted, which is in good agreement with the simulation result of $800 \mu \mathrm{V} / \mathrm{K}$. Theoretical calculations predict that the $\mathrm{ZrN} / \mathrm{ScN}$ structure can exhibit a $Z T$ of 1.5 at $1300 \mathrm{~K}$ assuming lateral momentum is conserved and that a $Z T$ of 3 is achievable if the lateral momentum is not conserved.
\end{abstract}

Key words: Thermoelectrics, metal/semiconductor interface

\section{INTRODUCTION}

The figure of merit, $Z T$, is a dimensionless parameter that determines the energy conversion efficiency of thermoelectric devices. $Z T$ is defined as $\sigma S^{2} T / \kappa$, where $\sigma$ is the electrical conductivity, $S$ is the Seebeck coefficient, $\kappa$ is the thermal conductivity, and $T$ is the absolute temperature. In recent years, new approaches have been under investigation to enhance $Z T$ by using low-dimensional thermoelectric materials.

At low dimensions, the Seebeck coefficient can be enhanced due to the abrupt change of the density of states $^{1}$ or as a result of hot electron filtering by potential barriers perpendicular to electron transport. $^{2}$ The thermal conductivity can be decreased by using interfaces to scatter phonons more effectively than electrons, for example, in the case of phonon

(Received July 8, 2008; accepted December 17, 2008;

published online January 19, 2009) blocking superlattices ${ }^{3}$ or embedded nanoparticles. $^{4,5}$

Many of the new thermoelectric materials having large $Z T$ values are limited to operating temperatures below $500^{\circ} \mathrm{C}$ due to their instabilities and device contact degradation at higher temperatures. Therefore, there is a need for high- $Z T$ materials for thermoelectric energy conversion that can operate under large temperature gradients from room temperature to about $1000^{\circ} \mathrm{C} .{ }^{6}$ Rocksalt nitride metal/ semiconductor materials are physically and chemically stable at very high temperatures. Here, we have investigated rocksalt nitride metal/semiconductor superlattices as novel thermoelectric metamaterials that utilize the thermoelectric enhancement of thermionic emission at heterointerfaces. The electron energy filtering effect of potential barriers is expected to increase the Seebeck coefficient while maintaining an adequate electrical conductivity due to the large density of states in the metal layers of the material. The 
abundant heterointerfaces along the transport direction also suppress the lattice thermal conductivity. Previous modeling of similar metal/semiconductor superlattices has shown that these superlattice structures can enhance the thermoelectric properties to achieve $Z T$ values in excess of 2 . $^{6}$

One of the major challenges in realizing metal/ semiconductor superlattices is identifying suitable material combinations that can be grown in the form of superlattices and maintain morphological stability at high operating temperatures. The considerations of melting point $\left(T_{\mathrm{m}}>2600^{\circ} \mathrm{C}\right)$, crystallographic compatibility, thermal expansion coefficients, and electronic properties have led to the selection of $(\mathrm{Zr}, \mathrm{W}) \mathrm{N} / \mathrm{ScN}$ as the desirable metal/ semiconductor combination.

In this paper, we report the growth and characterization of $\mathrm{ZrN} / \mathrm{ScN}$ samples. We apply a modified thermionic transport model to fit the experimental electrical conductivity. We then use the model to predict the performance of the structure at different temperatures and with different barrier heights.

\section{EXPERIMENT}

Coherent epitaxial multilayers of 4-nm-thick $\mathrm{ZrN}$ and 6-nm-thick $\mathrm{ScN}$ were grown on rocksalt $\mathrm{MgO}$ substrates by DC magnetron sputtering in an argon/ nitrogen ambient. Samples were etched to form pillars $1 \mu \mathrm{m}$ in height. Au/Cr layers $100 \mathrm{~nm}$ thick were subsequently deposited on the pillars to form the electrical contact layer.

The bulk properties of $\mathrm{ZrN}$ and $\mathrm{ScN}$ were characterized inside a thermostat under vacuum in the temperature range of $300 \mathrm{~K}$ to $800 \mathrm{~K}$. Figure 1 shows the experimental data for the bulk $\mathrm{ZrN}$ and $\mathrm{ScN}$. The electrical conductivity data along with the Hall measurement data was used to set the relaxation times for each layer in the modeling of $\mathrm{ZrN} / \mathrm{ScN}$ superlattices.

The electrical resistance of the $\mathrm{ZrN} / \mathrm{ScN}$ superlattices was measured in the cross-plane direction using the four-wire method in the range of $300 \mathrm{~K}$ to $475 \mathrm{~K}$. The electrical conductivity was extracted considering the geometry of the sample.

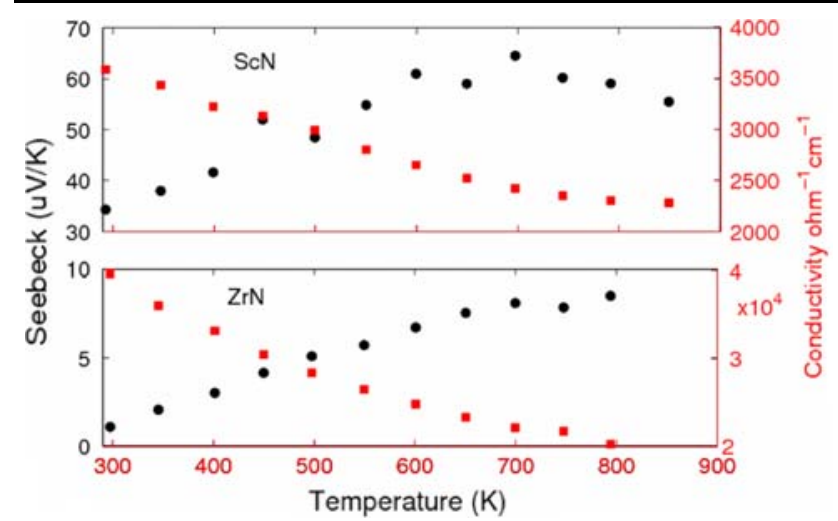

Fig. 1. Conductivity and Seebeck coefficient of bulk ScN and ZrN.

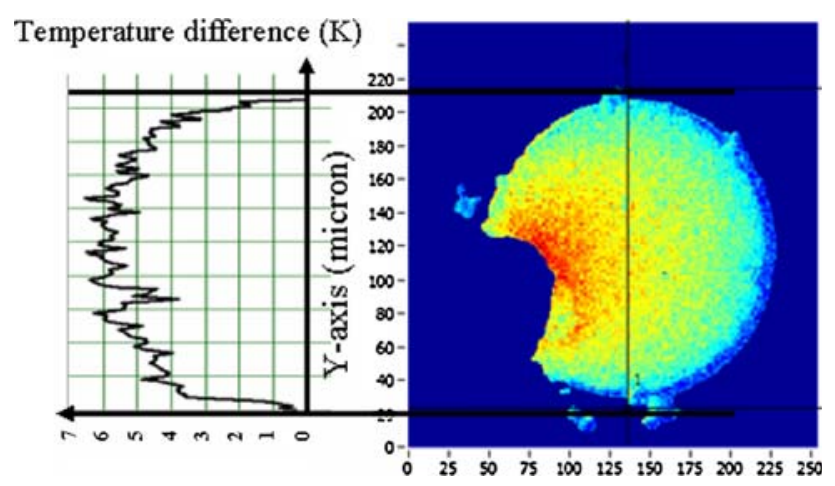

Fig. 2. Thermal imaging of the sample in the heating mode at room temperature and under an applied current of $150 \mathrm{~mA}$.

Cross-plane Seebeck coefficient was measured by combining the transient voltage measurement and the thermal imaging technique. A current pulse of $150 \mathrm{~mA}$ was applied to the sample in the cooling and heating modes. The transient voltage response was measured with an oscilloscope. The voltage signal consists of the resistive voltage and the Seebeck voltage due to Peltier cooling/heating and Joule heating. The resistive component of the total voltage drops instantaneously while the Seebeck voltage exhibits a slower decay time due to the thermal response of the thermoelectric device. The Seebeck voltage due to the Peltier effect was extracted by subtracting the Seebeck voltage amplitude in the cooling mode from the amplitude in the heating mode. Thermal imaging was utilized to measure the surface temperature of the top contact for an identical current amplitude. The thermal imaging results are shown in Fig. 2. A Seebeck coefficient of $820 \mu \mathrm{V} / \mathrm{K}$ is extracted from these data at room temperature.

\section{THEORETICAL MODEL}

A theoretical model was developed to calculate the thermoelectric properties of a superlattice structure. This model was based on a modified Boltzmann transport equation. ${ }^{7}$ A transmission coefficient due to the quantum-mechanical reflection coefficient was added to include the effect of quantum wells on the transport. The details of this formalism are explained in Ref. 7.

In order to calculate the transport coefficients of the superlattice structure, material properties of each layer are needed. The electronic relaxation times of each layer were obtained from the bulk electrical conductivity and Hall measurements. Eigenstates of the well were calculated from the Schrödinger equation. Band bending of the conduction bands was not taken into account. The Fermi level was calculated from the number of electrons in each layer and the thickness of the layers. Since the mean free path of the electrons is less than two period lengths of the superlattice, we only assume coupling of adjacent wells. Transmission 


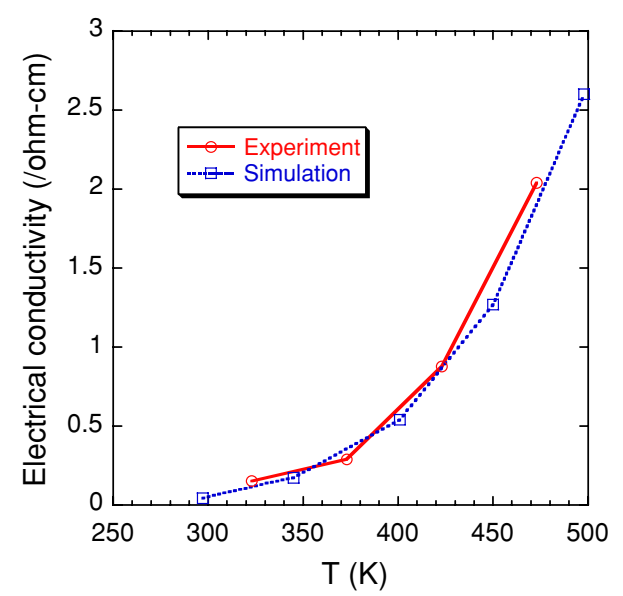

Fig. 3. Fit of experimental data of cross-plane electrical conductivity with the theoretical model. The fit results in an effective barrier height of $280 \mathrm{meV}$.

rates of the electrons were calculated using a doublebarrier tunneling model. These parameters were then used to calculate the number of electrons participating in the thermionic emission and the amount of energy that they carry. The electrical conductivity, Seebeck coefficient, and the electronic contribution to the thermal conductivity were then obtained. Finally, using an experimental value for the lattice thermal conductivity, the figure of merit was calculated for different barrier heights and temperatures.

In this simulation, effective masses of 0.2 and 1.5 were used for $\mathrm{ScN}$ and $\mathrm{ZrN}$, respectively. The carrier concentration in the metal layer was set to $5 \times 10^{22} \mathrm{~cm}^{-3}$ (from Hall data). The well width was $4 \mathrm{~nm}$ and the barrier width was $6 \mathrm{~nm}$. The barrier height of the metal/semiconductor junction was used as a fitting parameter in the model to fit the cross-plane electrical conductivity data. The fit of the experimental data with the numerical calculation indicates an effective conduction-band edge offset of $970 \mathrm{meV}$ with a Fermi level of $690 \mathrm{meV}$ and therefore an effective barrier height of $280 \mathrm{meV}$ (Fig. 3). At room temperature, the simulated Seebeck coefficient is $800 \mu \mathrm{V} / \mathrm{K}$, which is in good agreement with the experimental measurement. In the transport modeling, the lateral momentum was conserved when electrons pass heterointerfaces.

\section{DISCUSSION}

The electrical conductivity of the present sample is too low for thermoelectric applications near room temperature because its barrier height is too large. The barrier height can be adjusted in future samples by alloying or by varying the concentration of $\mathrm{N}$ in the metal layer. Figure 4 shows the results of the simulation assuming that the lateral momentum of the electrons is conserved when they pass the barrier. A constant lattice thermal conductivity of $1.8 \mathrm{~W} / \mathrm{m} \mathrm{K}$ was used in the calculation, which is the

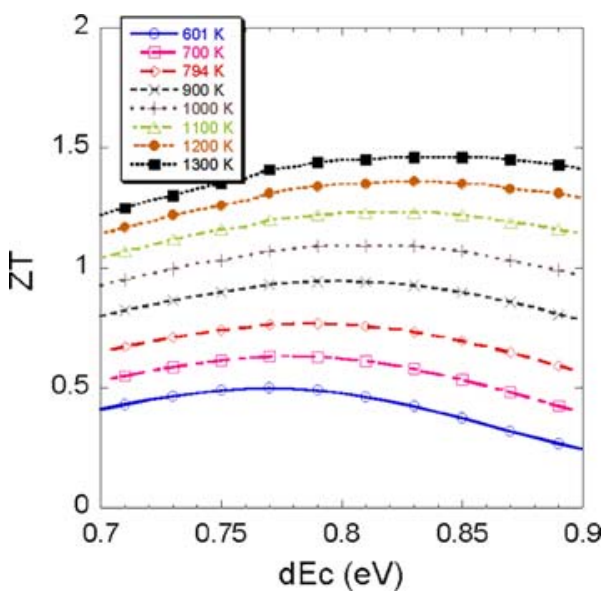

Fig. 4. Figure of merit versus conduction-band edge for $\mathrm{ZrN} / \mathrm{ScN}$ sample assuming lateral momentum is conserved.

measured value of the cross-plane lattice conductivity at room temperature. ${ }^{8}$ Since the lattice conductivity is expected to decrease with increasing temperature, the total thermal conductivity at higher temperatures is overestimated. This results in an underestimation of $Z T$. From the figure it can be seen that the optimum barrier height shifts slightly with the temperature. The effective bandedge offset changes from $750 \mathrm{meV}$ to $850 \mathrm{meV}$, which makes the effective barrier height $60 \mathrm{meV}$ to $160 \mathrm{meV}$. The figure of merit can increase to 1.5 at high temperatures. This simulation suggests that a lower barrier is needed for optimum enhancement.

It is known that a nonconserved lateral momentum can increase the number of carriers participating in the transport and the $Z T$ of the superlattice. ${ }^{9}$ This can be achieved by nanostructuring the interfaces and breaking the translational symmetry in the plane of the quantum wells. Figure 5 shows the improvement in the figure of

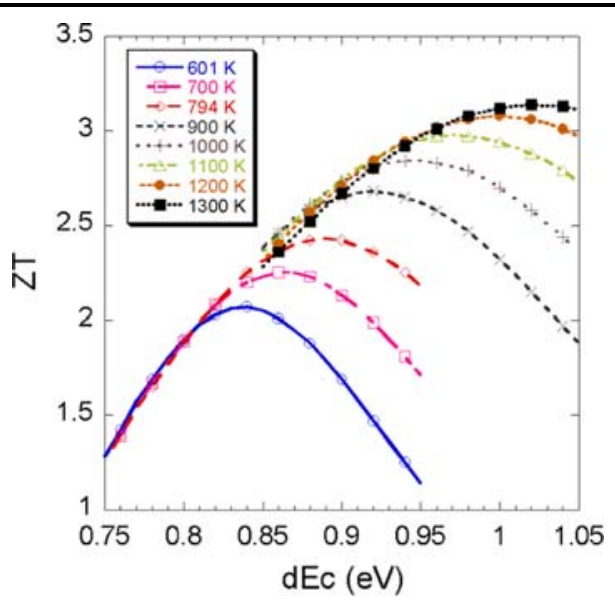

Fig. 5. Figure of merit versus conduction-band edge for $\mathrm{ZrN} / \mathrm{ScN}$ sample assuming lateral momentum is not conserved. 
merit if we assume a nonconserved lateral momentum at the junctions. The figure of merit can reach 3 at high temperatures. The optimum conductionband offset is higher for this case $(850 \mathrm{meV}$ to $1000 \mathrm{meV}$ ) and therefore the current material (band offset $\sim 970 \mathrm{meV}$ ) is close to the optimum and is expected to have a high figure of merit at high temperatures ( $Z T$ more than 2 for temperatures greater than $800 \mathrm{~K}$ ) if nanostructured interfaces are introduced.

\section{SUMMARY AND CONCLUSION}

A metal/semiconductor structure was designed and grown for high-temperature applications. It was shown experimentally and theoretically that the current transport in the structure is thermionic. Calculations predict that this structure can have an enhanced $Z T$. For nonconserved lateral momentum, material figure of merit can be as large as 3 at a temperature of $1200 \mathrm{~K}$. Nonconservation can be achieved by developing junctions with controlled roughness. ${ }^{10}$

\section{ACKNOWLEDGEMENT}

This work was supported by ONR MURI Thermionic Energy Conversion Center.

\section{OPEN ACCESS}

This article is distributed under the terms of the Creative Commons Attribution Noncommercial License which permits any noncommercial use, distribution, and reproduction in any medium, provided the original author(s) and source are credited.

\section{REFERENCES}

1. L.D. Hicks and M.S. Dresselhaus, Phys. Rev. B 47, 16631 (1993). doi:10.1103/PhysRevB.47.16631.

2. A. Shakouri and J.E. Bowers, Appl. Phys. Lett. 71, 1234 (1997). doi:10.1063/1.119861.

3. R. Venkatasubramanian, E. Siivola, T. Colpitts, and B. O'Quinn, Nature 413, 597 (2001). doi:10.1038/35098012.

4. T.C. Harman, P.J. Taylor, M.P. Walsh, and B.E. LaForge, Science 297, 2229 (2002). doi:10.1126/science.1072886.

5. J.M. Zide, D.O. Klenov, S. Stemmer, A.C. Gossard, G. Zeng, J.E. Bowers, D. Vashaee, and A. Shakouri, Appl. Phys. Lett. 87, 112102 (2005). doi:10.1063/1.2043241.

6. D.M. Rowe, ed., Thermoelectrics Handbook: Macro to Nano (Boca Raton: CRC, 2006), Chap. 43.

7. D. Vashaee and A. Shakouri, J. Appl. Phys. 95, 1233 (2004). doi:10.1063/1.1635992.

8. V. Rawat, Y.K. Koh, D.G. Cahill, and T.D. Sands, J. Appl. Phys., in press.

9. D. Vashaee and A. Shakouri, Phys. Rev. Lett. 92, 106103 (2004). doi:10.1103/PhysRevLett.92.106103.

10. Z.X. Bian and A. Shakouri, Appl. Phys. Lett. 88, 012012 (2006). doi:10.1063/1.2159574. 